\title{
Searches for exotic Higgs boson decays with the ATLAS and CMS experiments
}

\author{
Theodota Lagouri \\ on behalf of the ATLAS and CMS collaborations \\ Yale University \\ New Haven, CT USA \\ E-mail: theodota.lagouri@.cern.ch
}

\begin{abstract}
The observed Higgs boson offers an excellent opportunity to look for new physics at the LHC. Selected searches for exotic Higgs boson decays with the ATLAS and CMS experiments are presented. The Higgs boson decays to dark sector vector bosons, the Lepton Flavor Violating decays, the Flavor Changing Neutral Current decays, the decays to a light pseudo-scalar neutral Higgs boson of the NMSSM and the exotic Higgs boson decays to photon and missing transverse momentum signatures are described, and their Run-I results are given from both experiments. All the above searches for exotic Higgs boson decays present an alternative path to discovery of beyond Standard Model physics.
\end{abstract}

The European Physical Society Conference on High Energy Physics 22-29 July 2015

Vienna, Austria 


\section{Introduction}

The Higgs boson (indicated with $H$ or $h$ in the following) studied in various Standard Model (SM) channels by the ATLAS [1-2] and CMS [3-4] experiments at the LHC at CERN gives an excellent opportunity to look for new physics. The observed data was fitted to obtain Higgs boson coupling constants to SM fermions and gauge bosons. These fits constrain the branching fraction (B) of the Higgs boson to beyond the Standard Model (BSM) particles. Depending on the Higgs boson production cross section, the branching fraction $\mathrm{B}(h \rightarrow \mathrm{BSM})$ could be up to $\approx O(50 \%)$ [5]. Even a small BSM coupling can translate into a detectable signature since the predicted total width of the SM Higgs boson is small $(\approx 4 \mathrm{MeV})$. Therefore, the current precision of measurements of couplings to SM particles leaves ample room for BSM physics. An explicit search for (BSM) exotic Higgs boson decays presents an alternative opportunity for discovery of BSM physics and can provide the best window on dark matter.

Selected Run-I searches for exotic Higgs boson decays with the ATLAS and CMS experiments at the LHC are presented: the ATLAS analyses of Higgs boson decays to dark sector vector bosons [6], the analyses from ATLAS and CMS of the Lepton Flavor Violating (LFV) decays [7-8], the Flavor Changing Neutral Current (FCNC) decays [9-11], the decays to a light pseudo-scalar neutral Higgs boson of the NMSSM [12-14], and finally the exotic Higgs boson decays to photon and missing transverse momentum signatures are described [15-16] and their results are summarized. Finally, the conclusions of these searches are given at the end of this document.

All data referred to in this document was taken using $p p$ collisions at the LHC at a centreof-mass energy of $\sqrt{s}_{s}=8 \mathrm{TeV}$ with an integrated luminosity of about $20 \mathrm{fb}^{-1}$ and for a mass of the Higgs boson of $125 \mathrm{GeV}$, in some cases also data of $\sqrt{s}_{s}=7 \mathrm{TeV}$ were included.

\section{Search for new light gauge bosons in Higgs boson decays to four-lepton final states}

A search for Higgs bosons decaying to four leptons, either electrons or muons, via one or two light exotic gauge bosons $Z_{d^{\prime}} H \rightarrow Z Z_{d} \rightarrow 4 l$ or $H \rightarrow Z_{d} Z_{d} \rightarrow 4 l$ was performed with the ATLAS detector. Model-independent searches for dark sector states were performed [6].

For the $H \rightarrow Z Z_{d} \rightarrow 4 l$ search, in the absence of a significant signal, upper bounds were set on the relative branching ratio $\mathrm{R}_{\mathrm{B}}=\mathrm{BR}\left(H \rightarrow Z Z_{d} \rightarrow 4 l\right) / \mathrm{BR}(H \rightarrow 4 l)$ as function of the mass of the dark vector boson $m_{z_{d}}$. The $R_{B}$ limit at the $95 \%$ confidence level (CL) as a function of $m_{z_{d}}$ is shown in Fig. 1 (a). Observed and expected exclusion limits were estimated for the combination of all the four final states $(4 \mu, 4 \mathrm{e}, 2 \mu 2 \mathrm{e}, 2 \mathrm{e} 2 \mu)$. For relative branching ratios above 0.4 (observed) and 0.2 (expected), the entire mass range of $15<m_{z_{d}}<55 \mathrm{GeV}$ was excluded at the 95\% CL. 


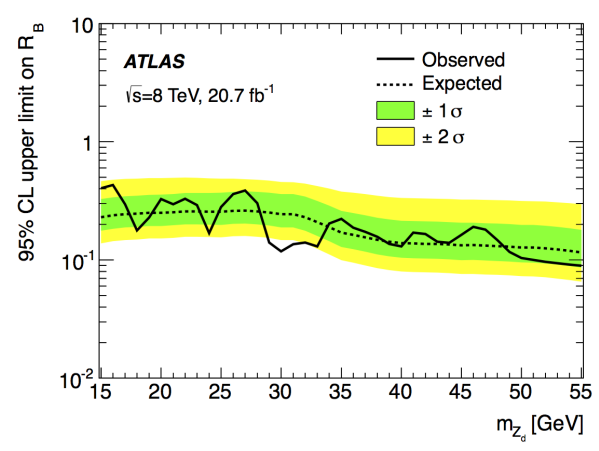

(a)

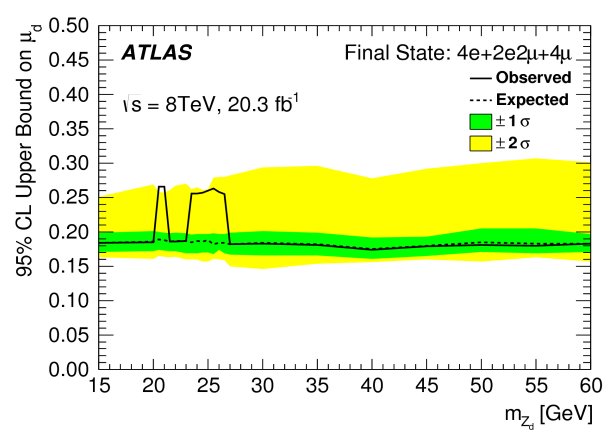

(b)

Figure 1: (a) The $95 \% \mathrm{CL}$ upper limits on the relative branching ratio, $R_{B}=\mathrm{BR}\left(H \rightarrow Z Z_{d} \rightarrow 4 l\right) / \mathrm{BR}(H \rightarrow 4 l)$ as a function of $m_{Z_{d}}$ [6]. (b) The $95 \%$ confidence level upper bound on the signal strength $\mu_{\mathrm{d}}=\sigma \times$ $\left.\mathrm{BR}\left(H \rightarrow \mathrm{Z}_{d} \mathrm{Z}_{d} \rightarrow 4 l\right)\right] /\left[\sigma \times \mathrm{BR}\left(H \rightarrow Z Z^{*} \rightarrow 4 l\right)\right]_{\mathrm{SM}}$ of $H \rightarrow Z_{d} Z_{d} \rightarrow 4 l$ in the combined $4 \mathrm{e}+2 \mathrm{e} 2 \mu+4 \mu$ final state, for $m_{H}=125 \mathrm{GeV}$ [6]. In both (a) and (b) the $\pm 1 \sigma$ and $\pm 2 \sigma$ expected exclusion regions are indicated in green and yellow, respectively.

The upper limits on $R_{B}$ led to upper limits on branching ratio of $H \rightarrow Z Z_{d} \rightarrow 4 l$ assuming the SM branching ratio of $H \rightarrow Z Z^{*} \rightarrow 4 l$ of $1.25 \times 10^{-4}$ [17-18]. They were found to be in the range $(1-9) \times 10^{-5}$ for $15<m_{Z_{d}}<55 \mathrm{GeV}$.

For the $H \rightarrow Z_{d} Z_{d} \rightarrow 4 l$ search, in the absence of a significant excess of events, upper bounds on the signal strength $\mu_{\mathrm{d}}=\sigma \times \mathrm{BR}\left(H \rightarrow \mathrm{Z}_{d} \mathrm{Z}_{d} \rightarrow 4 l\right) /\left[\sigma \times \mathrm{BR}\left(H \rightarrow Z Z^{*} \rightarrow 4 l\right)\right]_{\mathrm{SM}}$ were set for the mass range of $15<m_{Z_{d}}<60 \mathrm{GeV}$ using the combined $4 \mathrm{e}, 2 \mathrm{e} 2 \mu, 4 \mu$ final states. The 95\% CL upper bounds on $\mu_{\mathrm{d}}$ are shown in Fig. 1 (b) as a function of the hypothesized $m_{Z_{d}}$ for the combination of the three final states $4 \mathrm{e}, 2 \mathrm{e} 2 \mu$ and $4 \mu$. Assuming the SM Higgs boson production cross section and using $\mathrm{BR}\left(H \rightarrow Z Z^{*} \rightarrow 4 l\right)_{\mathrm{SM}}=1.25 \times 10^{-4}$, upper bounds on the branching ratio of $H \rightarrow Z_{d} Z_{d} \rightarrow 4 l$ were obtained. They were set in the range (2-3) $\times 10^{-5}$ at the $95 \% \mathrm{CL}$, for $15<m_{Z_{d}}<60 \mathrm{GeV}$.

\section{Search for Lepton-Flavour-Violating decays of the Higgs boson}

The presence of non-SM Higgs boson decays, like lepton-flavour-violating (LFV) decays, would serve as direct evidence for physics beyond the SM. The observation of the Higgs boson offers the possibility of sensitive direct searches for LFV Higgs boson decays.

A direct search for lepton-flavour-violating decays of a Higgs boson to a $\mu-\tau$ pair $(H \rightarrow$ $\mu \tau)$, was performed by CMS. The search was done in two channels, $H \rightarrow \mu \tau_{e}$ and $H \rightarrow \mu \tau_{h}$, where $\tau_{e}$ and $\tau_{h}$ are tau leptons reconstructed in the electronic and hadronic decay channels, respectively [7]. 
The signal variable studied was the collinear mass, $M_{c o l}$, in the $100<M_{c o l}<150 \mathrm{GeV}$ region. The signal strength was set according to the SM $H$ production cross section at $M_{H}=125$ $\mathrm{GeV}$ with $\mathrm{B}(H \rightarrow \mu \tau)=10 \%$. A slight excess of events with a significance of $2.4 \sigma$ was observed. The best fit branching fraction is $\mathrm{B}(H \rightarrow \mu \tau)=\left(0.84^{+0.39}{ }_{-0.37}\right) \%$. A constraint of $\mathrm{B}(H$ $\rightarrow \mu \tau)<1.51 \%$ at the $95 \% \mathrm{CL}$ was set. The limit was used to constrain the Yukawa couplings, $\left|Y_{\mu \tau}\right|+\left|Y_{\tau \mu}\right|<3.6 \times 10^{-3}$ and it is shown in Fig. 2 (a). It improves the previous bound by an order of magnitude.

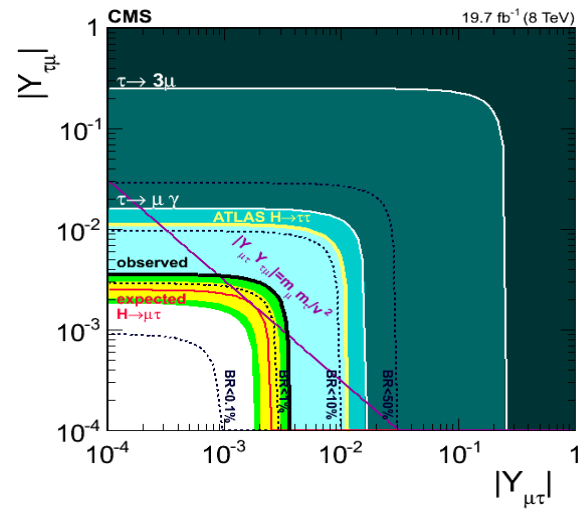

(a)

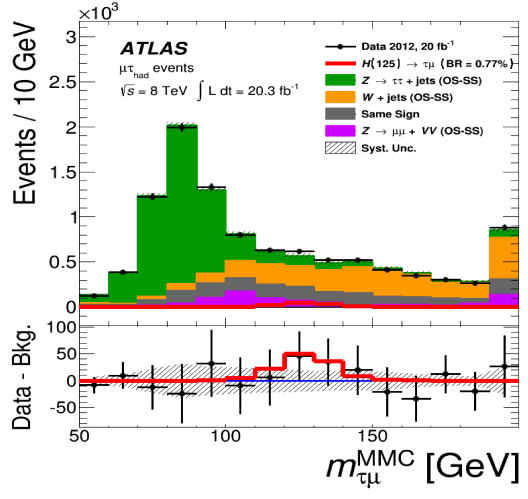

(b)

Figure 2: (a) Constraints on the flavour-violating Yukawa couplings, $\left|Y_{\mu \tau}\right|$ and $\left|Y_{\tau \mu}\right|$ [7]. The black dashed lines are contours of $\mathrm{B}(H \rightarrow \mu \tau)$ for reference. The expected limit (red solid line) with one sigma (yellow) and two sigma (green) bands, and observed limit (black solid line) are derived from the limit on $\mathrm{B}(H \rightarrow$ $\mu \tau)$ from the present analysis. The shaded regions are derived constraints from null searches for $\tau \rightarrow 3 \mu$ (dark green) and $\tau \rightarrow \mu \gamma$ (lighter green). The yellow line is the limit from a theoretical reinterpretation of an ATLAS $H \rightarrow \tau \tau$ search [19]. The light blue region indicates the additional parameter space excluded by ATLAS result. The purple diagonal line is the theoretical naturalness limit $Y_{i j} Y_{j i} \leq m_{i} m_{j} / v^{2}$. (b) Post-fit combined $m_{\mu \tau}{ }^{M M C}$ distribution obtained by adding individual distributions in SR1 and SR2 [8]. The grey band in the bottom panel plot illustrates the post-fit systematic uncertainties on the background prediction. The signal is shown assuming $\operatorname{Br}(H \rightarrow \mu \tau)=0.77 \%$, the central value of the best fit to $\operatorname{Br}(H \rightarrow$ $\mu \tau)$. The last bin of the distribution contains overflow events.

A direct search for LFV $H \rightarrow \mu \tau$ decays was also performed with the ATLAS detector [8]. The analysis was performed in the $H \rightarrow \mu \tau_{\text {had }}$ channel, where $\tau_{\text {had }}$ is a hadronically decaying $\tau-$ lepton. The presence of signal was inferred from an excess of data over the predicted background using the mass, $m_{\mu \tau}{ }^{M M C}$ (MMC [20]). The post-fit $m_{\mu \tau}{ }^{M M C}$ distributions in the combined distribution for signal regions (SR1 and SR2) is shown in Fig. 2 (b). A small excess of the data over the predicted background was found in the $120 \mathrm{GeV}<m_{\mu \tau}{ }_{M} M C<140 \mathrm{GeV}$ region in SR2, having a local significance of 2.2 standard deviations and a combined significance for both signal regions of 1.3 standard deviations. This corresponds to a best fit value for the branching fraction of $\operatorname{Br}(H \rightarrow \mu \tau)=(0.77 \pm 0.62) \%$. Due to the low significance of the observed excess of events, an upper limit on the LFV branching ratio $\operatorname{Br}(H \rightarrow \mu \tau)$ for a Higgs boson with $m_{H}=125 \mathrm{GeV}$ was set. The observed and the median expected upper limits at 
the $95 \%$ CL were found to be $1.85 \%$ and $\left(1.24^{+0.50}-0.35\right) \%$, respectively. This search places significantly more stringent constraints on $\operatorname{Br}(H \rightarrow \mu \tau)$ compared to earlier indirect estimates. The result of this analysis was found to be consistent with the one published by the one performed by the CMS Collaboration and described above.

\section{Search for Flavour-Changing-Neutral-Current decays}

The decay of a top quark to a Higgs boson plus an up-type $(c, u)$ quark would proceed via a flavour-changing neutral current (FCNC). According to the SM, FCNC processes are forbidden at tree level and very much suppressed at higher orders due to the Glashow-Iliopoulos-Maiani mechanism [21]. Observations of FCNC decays of the top quark would therefore provide a clear signal of new physics.

The FCNC $t \rightarrow q H$ decay, followed by $H \rightarrow \gamma \gamma$, has been searched for in a data set of proton-proton collisions recorded by the ATLAS experiment, consisting of $4.7 \mathrm{fb}^{-1}$ at $\sqrt{\mathrm{s}}=7 \mathrm{TeV}$ and $20.3 \mathrm{fb}^{-1}$ at $\sqrt{\mathrm{s}}_{\mathrm{s}}=8 \mathrm{TeV}$ [9].

The remaining top quark in the event was searched for in two final states: a bottom quark and either a hadronically decaying $\mathrm{W}$ boson or a leptonically decaying $\mathrm{W}$ boson. In the hadronic selection, which combined the 7 and $8 \mathrm{TeV}$ data, the diphoton mass distribution $m_{\gamma \gamma}$ was used as discriminating variable in the fit. The analysis in the leptonic selection was based on event counting.

The fitted branching ratio was found to be $\mathrm{B}=\left(0.22^{+0.31}-0.26\right) \%$. As no significant signal was found, limits on the $t \rightarrow c H$ and $t \rightarrow u H$, branching ratios were derived. The evolution of the signal confidence level CLs as a function of the branching fraction B for $t \rightarrow q H$ is shown in Fig. 3. The observed (expected) limit on B is $0.79(0.51) \%$ at the $95 \%$ CL.

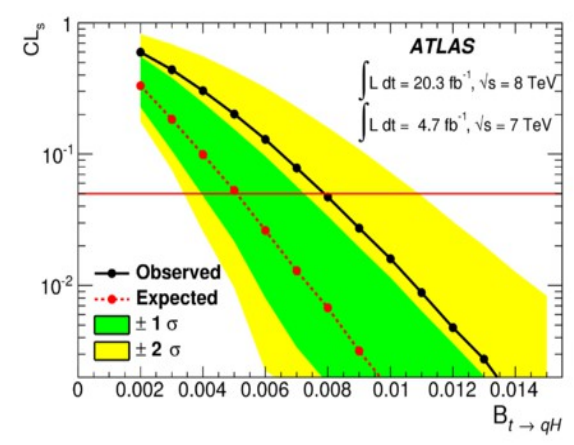

Figure 3: Evolution of CLs as a function of the branching fraction B of the $t \rightarrow q H$ decay for the observation of a signal at $125.5 \mathrm{GeV}$ (solid line) and the expectation in the absence of signal (dashed line) [9]. The 1 and $2 \sigma$ uncertainty bands around the expected curve are also shown.

An upper limit on the $\lambda_{t c H}$ coupling of 0.17 was obtained, with an expected value of 0.14 . As the analysis was equally sensitive to the $t \rightarrow u H$ and $t \rightarrow c H$ modes, the limit obtained on the couplings can be written as $\sqrt{ }\left(\lambda^{2}{ }_{t c H}+\lambda^{2}{ }_{t u H}\right)<0.17$ with an expectation of 0.14 .

A similar search for top quark decays via a flavor-changing neutral current was performed in CMS [10]. No significant excess of events was observed in the number of diphoton events in the hadronic and leptonic channels. An upper limit was set on the branching fraction for the 
signal production. Fig. 4 (a) shows the signal strength (r) at the $95 \% \mathrm{CL}$ as a function of the branching fraction $\mathrm{B}$ for $t \rightarrow c H$ by combining the hadronic and leptonic channels. An observed (expected) limit on the branching fraction B for $t \rightarrow c H$ was found $0.47 \%(0.71 \%)$ at the $95 \%$ CL. Similar results were presented for $\mathrm{t} \rightarrow u H$ samples. Fig. 4 (b) shows the result by combining the hadronic and leptonic channels. An observed (expected) limit on the branching fraction B for $t \rightarrow u H$ was found to be $0.42 \%(0.65 \%)$ at $95 \% \mathrm{CL}$.

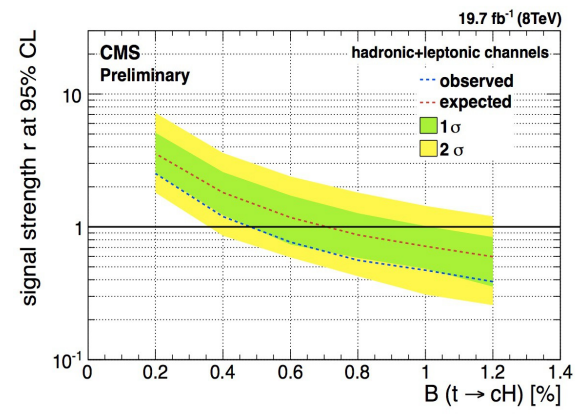

(a)

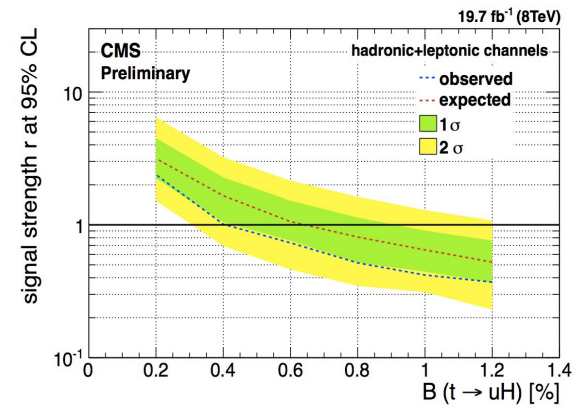

(b)

Figure 4: (a) Signal strength at the $95 \% \mathrm{CL}$ as a function of the branching fraction $\mathrm{B}$ for $t \rightarrow c H$ of the combined hadronic and leptonic channels [10]. (b) Signal strength at the $95 \% \mathrm{CL}$ as a function of the branching fraction B for $t \rightarrow u H$ of the hadronic and leptonic channels combined [10].

Based on the CMS inclusive multilepton analysis and a CMS 2HDM search involving lepton and photon final states, a limit at the $95 \% \mathrm{CL}$ on the branching ratio of the flavorchanging decay $t \rightarrow c H$ was published [11]. Given the good agreement between data and background, an upper limit was placed of $0.56 \%$ on $\mathrm{B}(t \rightarrow c H)$, consistent with the expected limit of $\left(0.65^{+0.29}-0.19\right) \%$. The observed limit corresponds to a bound on the left- and righthanded top-charm flavor violating Higgs Yukawa couplings of $\sqrt{ }\left(\left|\lambda_{t c}^{h}\right|^{2}+\left|\lambda_{c t}^{h}\right|^{2}\right)<0.14$.

\section{Higgs boson decays to light pseudo-scalar neutral Higgs boson in NMSSM}

A search for the pair production of new light bosons (denoted as "a $a_{1}$ " or "a"), each decaying into a pair of isolated muons, was performed with the CMS experiment at the LHC [12].

A range of new physics scenarios predict this decay topology, including the next-tominimal supersymmetric standard model (NMSSM) [22] and models with hidden (or dark) sectors [23-25]. The $\mathrm{a}_{1}$ boson can have a substantial branching fraction $\mathrm{B}\left(\mathrm{a}_{1} \rightarrow \mu^{+} \mu^{-}\right)$if its mass is within $2 m_{\mu}<m_{\mathrm{a} 1}<2 m_{\tau}$ [26-27]. A search for final states containing muon pairs provides sensitivity to models of this form.

One event was observed in the signal region, with $2.2 \pm 0.7$ events expected from the SM backgrounds. The 95\% CL upper limits from this search for the NMSSM scenarios are shown in Fig. 5. 


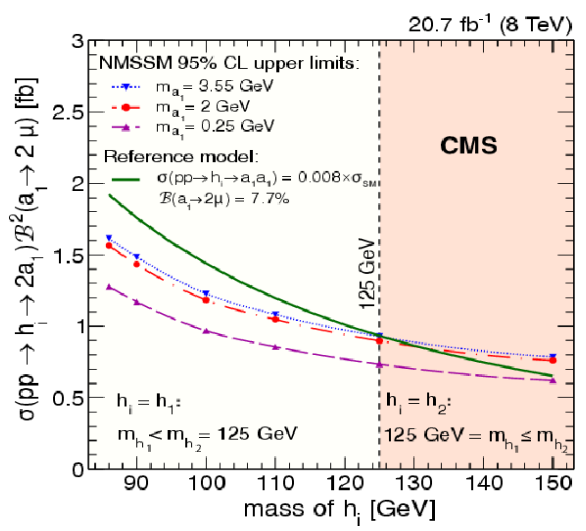

Figure 5: 95\% CL upper limits from the search for the NMSSM scenarios with $m_{\mathrm{a} 1}=0.25 \mathrm{GeV}$ (dashed curve), $m_{\mathrm{a} 1}=2 \mathrm{GeV}$ (dash-dotted curve) and $m_{\mathrm{a} 1}=3.55 \mathrm{GeV}$ (dotted curve) on $\sigma\left(p p \rightarrow h_{1 / 2} \rightarrow 2 \mathrm{a}_{1}\right) \mathrm{B}^{2}\left(\mathrm{a}_{1}\right.$ $\rightarrow 2 \mu)$ as a function of $m_{h 1}$ in the range $86<m_{h 1}<125 \mathrm{GeV}$ and of $m_{h 2}$ for $m_{h 2}>125 \mathrm{GeV}$ [12]. As an illustration, the limits are compared to the predicted rate (solid curve) obtained using a simplified scenario with $\sigma\left(p p \rightarrow h_{i} \rightarrow 2 \mathrm{a}_{1}\right)=0.008 \sigma_{\mathrm{SM}}$, which yields predictions for the rates of dimuon pair events comparable to the obtained experimental limits, and $\mathrm{B}\left(\mathrm{a}_{1} \rightarrow 2 \mu\right)=7.7 \%$. The chosen $\mathrm{B}\left(\mathrm{a}_{1} \rightarrow 2 \mu\right)$ is taken from [27] for $m_{\mathrm{a} 1}=2 \mathrm{GeV}$ and $\tan \beta=20$.

A search for the decay of a scalar Higgs boson to two pseudo-scalar "a" Higgs bosons ( $H$ $\rightarrow$ aa) in the $\mu \mu \tau \tau$ final state, in the context of the NMSSM was performed by the ATLAS experiment [13].

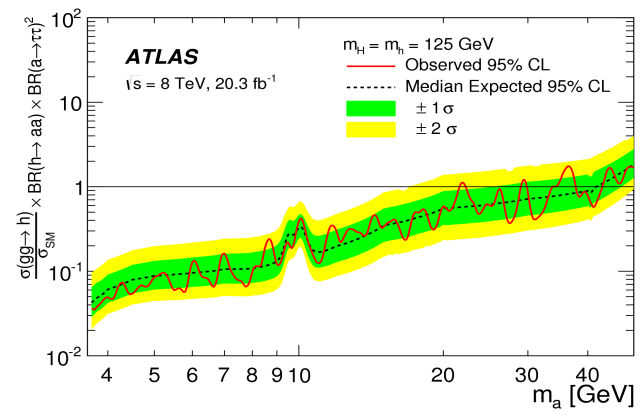

(a)

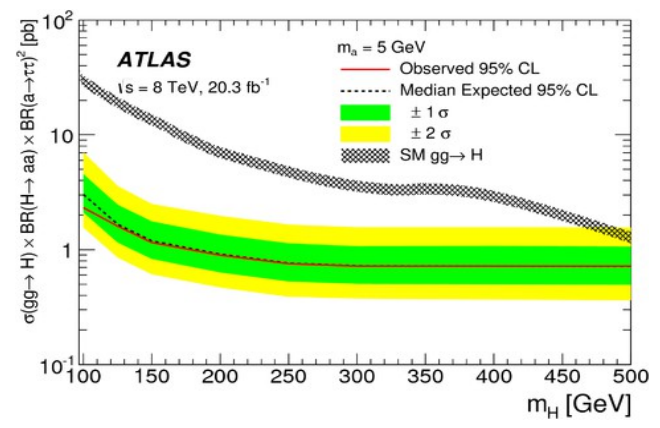

(b)

Figure 6: Observed (solid red) and expected (dashed black) limits with the expected \pm 1 and \pm 2 bands shown in green and yellow respectively. (a) Expected and observed limits on the rate $(\sigma(\mathrm{gg} \rightarrow h) \times \mathrm{BR}(h$ $\rightarrow$ aa)) relative to the SM Higgs boson gluon-gluon fusion production cross section (SM) as a function of $m_{\mathrm{a}}$ with $m_{H}$ set to $125 \mathrm{GeV}$ [13]. (b) The total rate $\left(\sigma(g g \rightarrow H) \times \mathrm{BR}(H \rightarrow\right.$ aa) $)$ as a function of $\mathrm{m}_{H}$ with $\mathrm{m}_{\mathrm{a}}$ set to $5 \mathrm{GeV}$, evaluated at $50 \mathrm{GeV}$ intervals from $m_{H}=100 \mathrm{GeV}$ to $500 \mathrm{GeV}$ [13]. The width of the black band indicates the theoretical uncertainty in the SM $g g \rightarrow H$ cross-section [28]. In both figures, the observed and expected limits have been scaled by an $O(1)$ parameter, $\mathrm{BR}(\mathrm{a} \rightarrow \tau \tau)^{2}$, to account for the branching ratios assumed in this analysis and facilitate reinterpretation of the results.

For the purposes of this analysis, the assumption of no coupling of the "a" boson to quarks was made. A scan of the dimuon invariant mass distribution from $3.7 \mathrm{GeV}$ to $50 \mathrm{GeV}$ showed 
no significant excess of data over SM backgrounds. With no evidence to support the NMSSM hypothesis, a 95\% CL limit was set. The Fig. 6 (a) shows the observed and expected limits on the rate $(\sigma(g g \rightarrow h) \times \mathrm{BR}(h \rightarrow$ aa $))$ relative to the SM Higgs boson gluon-gluon fusion production cross section (SM), as a function of $m_{\mathrm{a}}$ from $3.7 \mathrm{GeV}$ to $50 \mathrm{GeV}$ with $m_{H}$ set to 125 $\mathrm{GeV}$, with the most stringent limit placed at $3.5 \%$ for $\mathrm{m}_{\mathrm{a}}=3.75 \mathrm{GeV}$. Also in Fig. 6 (b) the total rate $(\sigma(\mathrm{gg} \rightarrow H) \times \mathrm{BR}(H \rightarrow \mathrm{aa}))$ is given as a function of $m_{H}$ with $m_{\mathrm{a}}$ set to $5 \mathrm{GeV}, m_{H}$ from 100 $\mathrm{GeV}$ to $500 \mathrm{GeV}$ and at $m_{H}=m_{h}=125 \mathrm{GeV}$. The observed 95\% CL upper limits on the production rate were consistent with the expected limit and were determined to be from $2.33 \mathrm{pb}$ to $0.72 \mathrm{pb}$.

Searches for new phenomena in events with at least three photons in the ATLAS detector were studied [14]. A model-independent search was performed for a Higgs boson decaying to four photons via two intermediate pseudoscalar "a" particles (for both the SM Higgs boson of $m_{h}=125 \mathrm{GeV}$ and for Higgs-like scalars of higher masses).

For the $H / h \rightarrow$ aa $\rightarrow 4 \gamma$ BSM signal scenario, the resonance search in the diphoton mass spectrum detected no significant excess of events above background. Upper limits (assuming kinematics associated only with gluon-gluon fusion Higgs boson production), were determined to be $\sigma / \sigma_{\mathrm{SM}} \times \mathrm{BR}(h \rightarrow \mathrm{aa}) \times \mathrm{BR}(\mathrm{a} \rightarrow \gamma \gamma)^{2}<1 \times 10^{-3}$, for $10 \mathrm{GeV}<m_{\mathrm{a}}<62 \mathrm{GeV}$. Additionally, limits were set for Higgs boson-like scalars with masses larger than $125 \mathrm{GeV}$. The results of these resonance searches are shown in Fig. 7 for the SM Higgs boson (a) and, as an example of a higher scalar mass, for $m_{H}=600 \mathrm{GeV}$ (b). Limits were found to be $\sigma_{H} \times \mathrm{BR}(H \rightarrow \mathrm{aa}) \times$ $\mathrm{BR}(\mathrm{a} \rightarrow \gamma \gamma)^{2}<0.02 \mathrm{pb}$ for $10 \mathrm{GeV}<m_{\mathrm{a}}<90 \mathrm{GeV}$ and $<0.001 \mathrm{pb}$ for higher $m_{\mathrm{a}}$ (up to $245 \mathrm{GeV}$ for the resonance search for $m_{H}=600 \mathrm{GeV}$ shown in Fig. 7 (b).

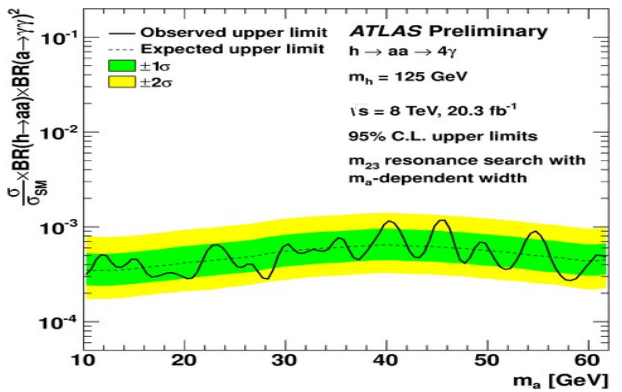

(a)

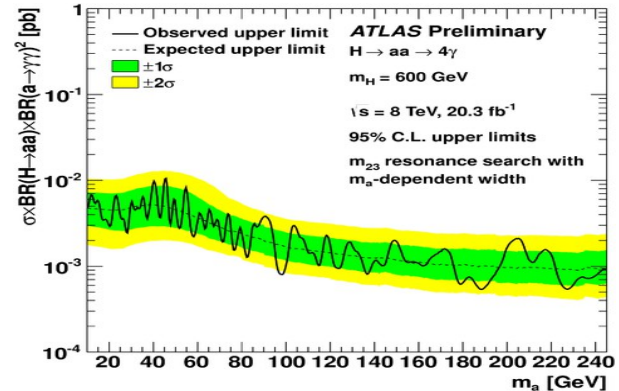

(b)

Figure 7: Upper lmits at the $95 \% \mathrm{CL}$, on (a) $\sigma / \sigma_{\mathrm{SM}} \times \mathrm{BR}(h \rightarrow \mathrm{aa}) \times \mathrm{BR}(\mathrm{a} \rightarrow \gamma \gamma)^{2}[14]$ and

(b) $\sigma_{H} \times \mathrm{BR}(H \rightarrow \mathrm{aa}) \times \mathrm{BR}(\mathrm{a} \rightarrow \gamma \gamma)^{2}$ [14]. Also shown are the $\pm 1 \sigma$ and $2 \sigma$ uncertainty bands resulting from the resonance search hypothesis tests, taking into account the statistical and systematic uncertainties from simulated signal samples which are used to determine signal efficiency and Gaussian resonance width due to detector resolution for each mass hypothesis. 


\section{Searches for exotic Higgs boson decays into undetectable particles and photons}

A search was performed in ATLAS for exotic Higgs boson decays to neutralinos and/or gravitinos in events with at least one photon, missing transverse momentum and two forward jets, a topology where vector boson fusion production was enhanced [15].

A cut-and-count experiment was performed and the total number of expected background and signal events in the control and signal regions were used to set limits on $\left(\sigma / \sigma_{\mathrm{SM}}\right) \times \mathrm{BF}(h \rightarrow$ NLSP + LSP). After all selections, the number of events observed agree with the expected SM backgrounds within uncertainties. The limits obtained were similar to or stronger than indirect limits from the Higgs boson coupling measurements, and were the first direct limits on these Higgs boson decays. The best-fit value of the signal yield corresponds to a BF of (11.6 \pm $10.9) \%$. The significance of the excess of observed data over the expected SM backgrounds was 1.1.

Fig. 8 shows the expected and observed limits on the $\left(\sigma / \sigma_{\mathrm{SM}}\right) \times \mathrm{BF}(h \rightarrow \mathrm{NLSP}+\mathrm{LSP})$ at the 95\% CL for the monophoton (a) and and diphoton (b) signal samples, obtained using the CLs method [29] with an Asimov approximation.

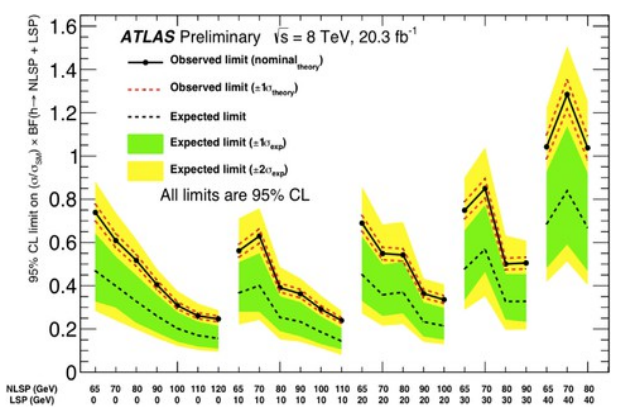

(a)

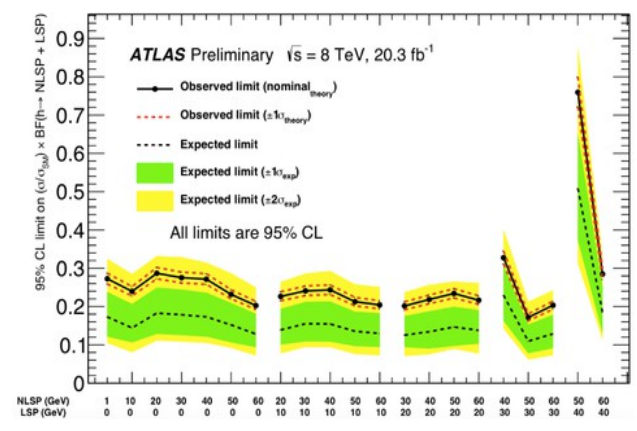

(b)

Figure 8: Observed and expected limits for various NLSP and LSP masses for (a) monophoton signals [15] and (b) diphoton signals [15]. The green and yellow bands show the \pm 1 and \pm 2 excursions of the expected limits respectively.

A similar search was also performed in CMS for exotic decays of a Higgs boson into undetectable particles and one or two isolated photons [16].

Higgs bosons produced in gluon-gluon fusion and in association with a $\mathrm{Z}$ boson were investigated, using models in which the Higgs boson decays into a gravitino and a neutralino or a pair of neutralinos, followed by the decay of the neutralino to a gravitino and a photon. The selected events were consistent with the background-only hypothesis, and limits were placed on the product of cross sections and branching fractions.

The $95 \% \mathrm{CL}$ upper limits on $(\sigma B) / \sigma_{\mathrm{SM}}$, where $\sigma_{\mathrm{SM}}$ is the production cross section for the SM Higgs boson, were evaluated for different neutralino $\left(\chi_{1}^{0}\right)$ mass values ranging from $1 \mathrm{GeV}$ to $120 \mathrm{GeV}$ for the individual searches and their combination and are shown in Fig. 9. An upper limit in the range of 7 to $13 \%$ was obtained. A $95 \%$ CL upper limit on the branching fraction of $10 \%$ is set for a neutralino mass of $95 \mathrm{GeV}$. 


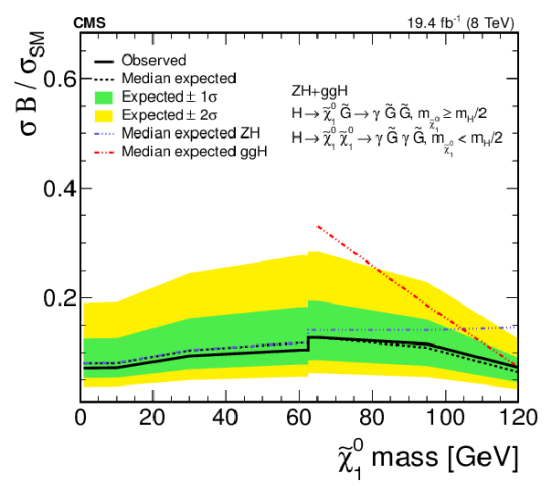

Figure 9: Expected and observed 95\% CL upper limits on $\sigma / \sigma_{\mathrm{SM}}$ for $m_{H}=125 \mathrm{GeV}$ as a function of $\tilde{\chi}_{1}{ }^{0}$ assuming the SM Higgs boson cross sections, for the $\mathrm{ZH}$ and $\mathrm{ggH}$ channels and their combination, with $B$ $\equiv B\left(H \rightarrow \tilde{\chi}_{1} \tilde{\chi}_{1}^{0}\right) B\left(\tilde{\chi}_{1}^{0} \rightarrow \tilde{\mathrm{G}}+\gamma\right)^{2}$ for $m \tilde{\chi}_{1}^{0}<m_{H} / 2$ and $B \equiv B\left(H \rightarrow \tilde{\chi}_{1}^{0} \tilde{\mathrm{G}}\right) B\left(\tilde{\chi}_{1}{ }^{0} \rightarrow \tilde{\mathrm{G}}+\gamma\right)$ for $m \tilde{\chi}_{1}{ }^{0} \geq m_{H} / 2$ [16].

Expected and observed limits are also shown for the decay of possible heavier scalar Higgs bosons as a function of the Higgs boson mass in Fig. 10.

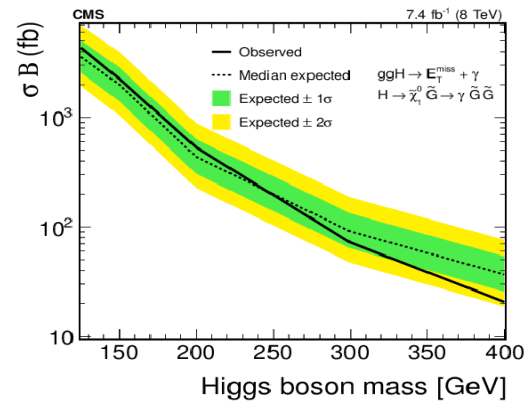

(a)

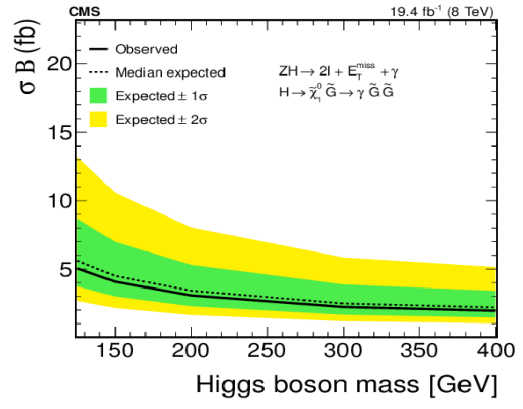

(b)

Figure 10: Expected and observed $95 \%$ CL upper limits on $\sigma_{g g \rightarrow H}$ as a function of the Higgs boson mass with $\tilde{\chi}_{1}^{0}=m_{H}-30 \mathrm{GeV}$ in the (a) $g g H$ channel [16] and (b) $Z H$ channel [16].

\section{Conclusions}

Searches for exotic Higgs bosons decays performed in various channels and with different strategies in the ATLAS and CMS experiments at the LHC were presented. No evidence of BSM physics has been found so far. The observation of the SM-like Higgs boson has already excluded large regions of the parameter phase space. However, there is still space left for BSM models to be compatible with the observed Higgs boson. Many exotic Higgs boson analyses with Run-I data from pp collisions at $\sqrt{\mathrm{s}}_{\mathrm{s}}=8 \mathrm{TeV}$ are still ongoing and are going to be published soon. The Run-II data from $p p$ collisions at $\sqrt{\mathrm{s}}_{\mathrm{s}}=13 \mathrm{TeV}$ will enhance the discovery potential of exotic Higgs boson decay searches and new challenges for the corresponding analyses have to be faced. 


\section{References}

[1] ATLAS Collaboration, JINST 3 S08003 (2008)!ل

[2] ATLAS Collaboration, Measurements of the Higgs boson production and decay rates and coupling strengths using pp collision data at $\sqrt{s}_{s}=7$ and $8 \mathrm{TeV}$ in the ATLAS experiment, [arXiv:1507.04548 [hepex]], submitted to Eur. Phys. J. C.

[3] CMS Collaboration, JINST 3 S08004 (2008).

[4] CMS Collaboration, Precise determination of the mass of the Higgs boson and tests of compatibility of its couplings with the standard model predictions using proton collisions at 7 and 8 TeV, Eur. Phys. J. C 75 (2015) 212, [arXiv:1412.8662 [hep-ex]].

[5] D. Curtin et al., Exotic Decays of the 125 GeV Higgs Boson, [arXiv:1312.4992 [hep-ph]].

[6] ATLAS Collaboration, Search for new light gauge bosons in Higgs boson decays to four-lepton final states in pp collisions at $\sqrt{s}=8 \mathrm{TeV}$ with the ATLAS detector at the LHC, [arXiv:1505.07645 [hep-ex]], accepted for publication in $P R D$.

[7] CMS Collaboration, Search for lepton-flavour-violating decays of the Higgs boson [arXiv:1502.07400 [hep-ex]], CMS-PAS-HIG-14-005, submitted to Phys. Lett. B.

[8] ATLAS Collaboration, Search for lepton-flavour-violating $H \rightarrow \mu \tau$ decays of the Higgs boson with the ATLAS detector, [arXiv:1508.03372 [hep-ex]], submitted to JHEP.

[9] ATLAS Collaboration, Search for top quark decays $t \rightarrow q H$ with $H \rightarrow \gamma \gamma$ using the ATLAS detector, JHEP 06 (2014) 008.

[10] CMS Collaboration, Search for top quark decays $t \rightarrow q H$ with $H \rightarrow \gamma \gamma$ in pp collisions at $\sqrt{s}=8 \mathrm{TeV}$, CMS-PAS-TOP-14-019.

[11] CMS Collaboration, Searches for heavy Higgs bosons in two-Higgs-doublet models and for t to ch decay using multilepton and diphoton final states in pp collisions at $8 \mathrm{TeV}$, Phys. Rev. D 90 (2014) 112013, [arXiv:1410.2751 [hep-ex]].

[12] CMS Collaboration, A search for pair production of new light bosons decaying into muons, [arXiv:1506.00424 [hep-ex]], submitted to Phys. Lett. B.

[13] ATLAS Collaboration, Search for Higgs bosons decaying to a in the $\mu \mu \tau \tau$ final state in pp collisions at $\sqrt{ } \mathrm{s}=8 \mathrm{TeV}$ with the ATLAS experiment, Phys. Rev. D 92, 052002 (2015), [arXiv:1505.01609 [hep-ex]].

[14] ATLAS Collaboration, Search for new phenomena in events with at least three photons collected in pp collisions at $\sqrt{s}=8 \mathrm{TeV}$ with the ATLAS detector, [arXiv:1509.05051 [hep-ex]], submitted to Eur. Phys. J. C.

[15] ATLAS Collaboration, Search for exotic Higgs-boson decays in events with at least one photon, missing transverse momentum, and two forward jets produced in $\sqrt{s}=8 \mathrm{TeV}$ pp collisions with the ATLAS detector, ATLAS-CONF-2015-001.

[16] CMS Collaboration, Search for exotic decays of a Higgs boson into undetectable particles and photons, arXiv:1507.00359 [hep-ex], CMS-HIGG-14-025, submitted to Phys. Lett. B.

[17] LHC Higgs cross section working group et al., Handbook of LHC Higgs cross sections: 1. Inclusive observables, CERN-2011-002 (2011), [arXiv: 1101.0593 [hep-ph]].

[18] LHC Higgs cross section working group et al., Handbook of LHC Higgs Cross Sections: 2.Differential distributions, CERN-2012-002 (2012), [arXiv: 1201.3084 [hep-ph]]. 
[19] R. Harnik, J. Kopp, and J. Zupan, Flavor violating Higgs decays, JHEP 03 (2013) 026, doi:10.1007/JHEP03(2013)026, [arXiv:1209.1397 [hep-ex]].

[20] A. Elagin, P. Murat, A. Pranko, and A. Safonov, A New Mass Reconstruction Technique for Resonances Decaying to di-tau, Nucl. Instrum. Meth. A 654 (2011) 481-489, [arXiv:1012.4686 [hep-ex]].

[21] S. L. Glashow, J. Iliopoulos, and L. Maiani, Weak Interactions with Lepton-Hadron Symmetry, Phys. Rev. D 2 (1970) 1285.

[22] U. Ellwanger, M. Rausch de Traubenberg, and C. A. Savoy, Phenomenology of supersymmetric models with a singlet, Nucl. Phys. B 492 (1997) 21, doi:10.1016/S0550-3213(97)80026-0, [arXiv:hep$\mathrm{ph} / 9611251]$.

[23] N. Arkani-Hamed, D. P. Finkbeiner, T. R. Slatyer, and N. Weiner, A theory of dark matter, Phys. Rev. D 79 (2009) 015014, doi:10.1103/PhysRevD.79.015014, [arXiv:0810.0713 [hep-ph]].

[24] M. Baumgart et al., Non-abelian dark sectors and their collider signatures, JHEP 04 (2009) 014, doi:10.1088/1126-6708/2009/04/014, [arXiv:0901.0283 [hep-ph]].

[25] A. Falkowski, J. T. Ruderman, T. Volansky, and J. Zupan, Hidden Higgs decaying to lepton jets, JHEP 05 (2010) 077, doi:10.1007/JHEP05(2010)077, [arXiv:1002.2952 [hep-ph]].

[26] A. Belyaev et al., LHC discovery potential of the lightest NMSSM Higgs boson in the $h_{1} \rightarrow a_{1} a_{1} \rightarrow$ $4 \mu$ channel, Phys. Rev. D 81 (2010) 075021, doi:10.1103/PhysRevD.81.075021, [arXiv:1002.1956 hep$\mathrm{ph}]$.

[27] R. Dermisek and J. F. Gunion, New constraints on a light CP-odd Higgs boson and related NMSSM ideal Higgs scenarios, Phys. Rev. D 81 (2010) 075003, doi:10.1103/PhysRevD.81.075003, [arXiv:1002.1971 [hep-ph]].

[28] LHC Higgs cross section working group et al., Handbook of LHC Higgs Cross Sections: 3. Higgs properties, CERN-2013-004 (2013), arXiv:1307.1347 [hep-ph].

[29] A. L. Read, Presentation of search results: The CL(s) technique, J.Phys. G28 (2002) 2693-2704. 